\title{
Activin A Expressed in Rheumatoid Synovial Cells Downregulates TNFa-Induced CXCL10 Expression and Osteoclastogenesis
}

\author{
Tatsuomi Kuranobu Sho Mokuda Katsuhiro Oi Tadahiro Tokunaga \\ Kazutoshi Yukawa Hiroki Kohno Yusuke Yoshida Shintaro Hirata \\ Eiji Sugiyama \\ Department of Clinical Immunology and Rheumatology, Hiroshima University Hospital, Hiroshima, Japan
}

\section{Keywords}

Rheumatoid arthritis - Activin A - CXCL10 .

Osteoclastogenesis

\begin{abstract}
Objective: Activin A is known to be highly expressed in rheumatoid synovium. In the present study, we investigated the effect of inflammatory cytokines on activin A production and its role in rheumatoid inflammation using freshly prepared rheumatoid synovial cells (fresh-RSC). Methods: Fresh-RSC from patients with rheumatoid arthritis were obtained and stimulated with multiple cytokines for activin A production. Gene expression levels of activin A and inflammatory cytokines were determined by quantitative PCR (qPCR) analysis. An enzyme-linked immunosorbent assay (ELISA) was used to measure activin A and CXCL10 in culture supernatants. The osteoclasts generated from human peripheral monocytes by RANKL stimulation were identified by tartrate-resistant acid phosphatase staining and bone resorption assay using Osteo plate. The expression levels of NFATc1 and cathepsin $\mathrm{K}$, critical intracellular proteins for osteoclastogenesis, were determined by Western blotting. Results: Activin A produc-
\end{abstract}

tion in fresh-RSC was markedly enhanced by the synergistic effect of TGF- $\beta_{1}$ with inflammatory cytokines, including TNFa, IL-1 $\beta$, and IL-6. Activin A inhibited TNFa-induced CXCL10, an important chemoattractant for pathogen-activated T cells and monocytes of osteoclast precursors, but it did not affect the expression of inflammatory cytokines and chemokines. In addition, activin A directly inhibited the expression of NFATc1 and cathepsin K, as well as osteoclast formation in human samples. Conclusion: Our data indicated that TGF- $\beta_{1}$ is involved in the expression of activin $A$ at inflamed joints. Activin A mainly exerts an anti-inflammatory action, which prevents joint damage via the regulation of CXCL10 and osteoclastogenesis.

(c) 2020 S. Karger AG, Basel

\section{Introduction}

Rheumatoid arthritis (RA) is a chronic inflammatory disease with hyperplasia of synovial cells and infiltration of inflammatory cells at the inflamed joint, and persistent inflammation results in progressive destruction of the articular cartilage and the subchondral bone $[1,2]$. Al-

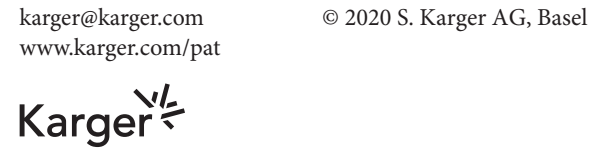


though the bone erosion of inflammatory joints is caused by osteoclast progenitor cells and mature osteoclasts [3, 4], a variety of cytokines and growth factors involved in the inflammatory process play important roles in the differentiation of osteoclasts $[5,6]$.

Activin A, a homodimer structurally composed of 2 $\beta A$ subunits $(\beta A \beta A)$, is expressed in a number of tissues including ovaries, pituitary gland, brain, placenta, and bone marrow [7]. Interestingly, activin A is also expressed by cells involved in rheumatoid inflammation, such as bone marrow macrophages, monocytes, osteoblasts, osteoclasts, and endothelial cells [7-11]. Additionally, activin A is highly expressed in the inflamed synovium of patients with RA $[9,10]$. Activin A is reported to be expressed in fibroblast-like synoviocytes (FLS) of the lining and sublining layers, and in macrophages of the active rheumatoid synovium [10]. Furthermore, the activin A level in the synovial fluid of RA patients has been shown to be higher than that of systemic lupus erythematosus or osteoarthritis [12].

The pathophysiological role of activin A in RA inflammation remains controversial. In murine models, administration of activin A induces the release of IL-6 [13, 14], whereas neutralizing activin A modulates the release of TNFa, IL-1 $\beta$, and IL-6 [15]. In addition, activin A can regulate human macrophage polarization, directing them toward an inflammatory M1 phenotype [16]. On the other hand, activin $\mathrm{A}$ has been reported to inhibit the production of MMP-3 [17] and inflammatory cytokines such as IL-1 $\beta$, IL-6, and IL-18 in a variety of cell types [18-20].

The functional role of activin A in osteoclastogenesis also remains unclear. Activin A has been shown to stimulate RANKL-induced osteoclast differentiation. Inhibition of activin A by its soluble receptor can inhibit osteoclast differentiation $[8,21,22]$. In contrast, activin $A$ has been shown to act as a local regulator during osteoclast development by exerting a dominant negative effect on the motility and lifespan of osteoclasts [23]. Furthermore, activin $\mathrm{A}$ acts as a negative regulator of osteoclast motility via activation of SMAD2 and AKT1 [23].

These conflicting data prompted us to clarify the regulation of expression and the pathophysiological role of activin $\mathrm{A}$ in RA. In the present study, to understand the role of activin A in osteoclastogenesis, we evaluated the effect of inflammatory cytokines on activin A production in freshly prepared rheumatoid synovial cells (freshRSC). In addition, we examined the effect of activin A on cytokine expression in fresh RSC and RANKL-induced osteoclastogenesis in human monocytes.

Activin A-Regulated Synovial CXCL10

Expression

\section{Materials and Methods}

\section{Preparation of Fresh-RSC}

A total of 6 patients with RA diagnosed based on the revised 1987 American College of Rheumatology criteria for RA were recruited. Synovial tissues were collected from 6 patients who underwent arthroplastic joint surgery and synovectomy.

Synovia obtained from RA patients were aseptically dissected from the surrounding tissues, minced, and enzymatically digested with $2 \mathrm{mg} / \mathrm{mL}$ Clostridium collagenase (Wako Pure Chemical Industries, Ltd, Osaka, Japan) and 5-10 $\mu \mathrm{g} / \mathrm{mL}$ deoxyribonuclease 1 (Sigma Chemical Co., St. Louis, MO, USA) for 2-3 h. After digestion, the resulting single cell suspension was washed, filtered through sterile gauze and nylon mesh, washed thoroughly again, and finally resuspended in Dulbecco's modified Eagle's medium (DMEM) supplemented with $10 \%$ heat-inactivated fetal calf serum. The cells were then cultured overnight to allow the cells to adhere to the culture plate. After washing the plate to remove nonadherent cells, the remaining adherent cells were cultured with the indicated cytokines, and adherent cells of 2-3 passages were further used in this study. The recombinant human cytokines used in this study were as follows: IL- $1 \beta, T N F \alpha$, IL-6, IL-6R $\alpha$, IL-17A, M-CSF (all BioLegend, San Diego, CA, USA), TGF- $\beta_{1}$, activin A, and RANKL (all Wako Pure Chemical Industries).

\section{Preparation of Peripheral Blood Monocytes}

Mononuclear cells from the peripheral blood of healthy donors were separated by Lympholyte ${ }^{\circledR}-\mathrm{H}($ Cedarlane, NC) density gradient centrifugation. Highly purified monocytes were separated using MACS microbeads (Pan Monocyte Isolation Kit ${ }^{\circledR}$, Miltenyi Biotec GmbH, Bergisch Gladbach, Germany). All procedures were performed according to the manufacturers' instructions. More than $92 \%$ of the isolated cells were found to be CD14+, as determined by flow cytometry.

\section{qPCR Analysis}

Cells were lysed, and total RNA was isolated using a NucleoSpin ${ }^{\circledR}$ RNA kit (Takara-Bio Japan). Total RNA (200 ng) was then reverse transcribed to $\mathrm{CDNA}$ using a PrimeScript ${ }^{\mathrm{TM}} \mathrm{RT}$ reagent kit with gDNA Eraser (Takara-Bio, Japan). qPCR was then performed using Brilliant III ultrafast SYBR Green qPCR master mix (Agilent Technologies, CA) with the CFX connect real-time PCR detection system (Bio-Rad Laboratories, Inc.). PCR amplification was performed for $40 \mathrm{cycles}$ at $95^{\circ} \mathrm{C}$ for $5 \mathrm{~s}$ and $60^{\circ} \mathrm{C}$ for $10 \mathrm{~s}$. The primers used in this study are shown in Table 1 . Gene expression was normalized to $\beta$-actin for each sample.

\section{ELISA for Activin A and CXCL10}

Activin A and CXCL10 concentrations in culture supernatant were measured using a sandwich ELISA kit (R\&D Systems, Minneapolis, MN, USA) according to the manufacturer's instructions.

\section{In vitro Osteoclastogenesis}

Peripheral blood monocytes (PBMs) were cultured in a 96-well tissue culture plate at a concentration of $2 \times 10^{4}$ cells/well. Cultures were maintained in $200 \mu \mathrm{L}$ of $\alpha$-minimal essential medium containing 10\% fetal bovine serum for 7-10 days with M-CSF (BioLegend) and RANKL (Wako Pure Chemical Industries) in the presence or absence of activin A (Wako Pure Chemical Industries). The cells were pretreated with M-CSF and/or activin A for $24 \mathrm{~h}$, 
Table 1. Primers used for real-time PCR

\begin{tabular}{|c|c|c|}
\hline Gene & Sense & Antisense \\
\hline Activin A & $5^{\prime}$-GAGAATTTGCTGAAGAGGAG-3' & 5'-CAAAAGTTGTTGTGATTGCC-3' \\
\hline$I L-1 \beta$ & $5^{\prime}$-CTAAACAGATGAAGTGCTCC-3' & $5^{\prime}$-GGTCATTCTCCTGGAAGG-3' \\
\hline$T N F \alpha$ & $5^{\prime}$-AGGCAGTCAGATCATCTTC-3' & $5^{\prime}$-TTATCTCTCAGCTCCACG-3' \\
\hline$I L-6$ & $5^{\prime}$-GCAGAAAAAGGCAAAGAATC-3' & $5^{\prime}$-CTACATTTGCCGAAGAGC-3' \\
\hline$I L-10$ & $5^{\prime}$-GCCTTTAATAAGCTCCAAGAG-3' & $5^{\prime}$-ATCTTCATTGTCATGTAGGC-3' \\
\hline$I L-12 A$ & $5^{\prime}$-AAGACCTCTTTTATGATGGC-3' & 5'-CATTCATGGTCTTGAACTCC-3' \\
\hline$I L-23 A$ & $5^{\prime}$-AGATAAATCTACCACCCCAG-3' & $5^{\prime}$-CACATGTCAGTCAGTATTGG-3' \\
\hline CXCL5 & $5^{\prime}$-ATTTGTCTTGATCCAGAAGC-3' & $5^{\prime}$-TCAGTTTTCCTTGTTTCCAC-3' \\
\hline CXCL8 & 5'-GTTTTTGAAGAGGGCTGAG-3' & 5'-TTTGCTTGAAGTTTCACTGG-3' \\
\hline CXCL9 & $5^{\prime}$-AGGTCAGCCAAAAGAAAAAG-3' & $5^{\prime}$-TGAAGTGGTCTCTTATGTAGTC-3' \\
\hline CXCL10 & $5^{\prime}$-AAAGCAGTTAGCAAGGAAAG-3' & $5^{\prime}$-TCATTGGTCACCTTTTAGTG-3' \\
\hline CCL2 & 5'-AGACTAACCCAGAAACATCC-3' & $5^{\prime}$-ATTGATTGCATGTGGCTG-3' \\
\hline CCL3 & $5^{\prime}$-TCTCTGCAACCAGTTCTC-3' & $5^{\prime}$-AATTCTGTGGAATCTGCC-3' \\
\hline CCL5 & 5'-AAGTCTCTAGGTTCTGAGC-3' & 5'-TTTTATGGTTGCATTGAGAAC-3' \\
\hline CCL20 & $5^{\prime}$-TATATTGTGCGTCTCCTCAG-3' & $5^{\prime}$-GCTATGTCCAATTCCATTCC-3' \\
\hline$\beta$-Actin & $5^{\prime}$-GACGACATGGAGAAAATCTG-3' & 5'-ATGATCTGGGTCATCTTCTC-3' \\
\hline
\end{tabular}

followed by the addition of RANKL to the culture medium. Every 3 days, half of the culture medium was replaced with fresh medium containing cytokines and chemicals.

\section{Tartrate-Resistant Acid Phosphatase Staining}

The cells were fixed, and tartrate-resistant acid phosphatase (TRACP) staining was performed using an acid phosphatase, leukocyte (TRACP) staining kit (Sigma-Aldrich Co.) according to the manufacturer's instructions. Cells that stained dark red were considered as differentiated osteoclast-like cells, and cells with $\geq 3 \mathrm{nu}$ clei were designated as multinucleated cells. TRACP-positive, multinucleated cells were counted on 96-well plates with a light microscope (EVOS ${ }^{\circledR}$ XL Core Cell Imaging System, Thermo Fisher Scientific, Japan).

\section{In vitro Assays for Osteoclast Resorption}

To measure bone-resorptive function, PBMs were cultured at a density of $2 \times 10^{4}$ cells/well on an Osteo plate (Corning, New York, NY, USA). The Osteo plate comprises a surface made of an inorganic crystalline calcium phosphate coating that mimics living bone material for 7-10 days. After culturing, the adherent cells were lysed by osmotic shock and stained with $5 \%$ silver nitrate (Sigma-Aldrich) in PBS to visualize the resorption area under an EVOS microscope using ImageJ software (National Institutes of Health, Bethesda, MD, USA).

\section{Western Blotting}

PBMs cultured with RANKL and M-CSF with or without activin A were washed with PBS, and protein was extracted using a radioimmunoprecipitation assay buffer $(50 \mathrm{~mm}$ Tris- $\mathrm{HCl}, \mathrm{pH} 8.0$, $150 \mathrm{~mm}$ sodium chloride, $0.5 \mathrm{w} / \mathrm{v} \%$ sodium deoxycholate, $0.1 \mathrm{w} / \mathrm{v} \%$ SDS, and $1.0 \mathrm{w} / \mathrm{v} \%$ NP-40 substitute; Wako Pure Chemical Industries). Total cell lysates were obtained by centrifugation at 12,000 $\mathrm{rpm}$ for $10 \mathrm{~min}$ at $4{ }^{\circ} \mathrm{C}$. Protein levels were determined, and equal protein levels were adjusted by the Pierce ${ }^{\mathrm{TM}}$ BCA protein assay (Thermo Fisher Scientific) according to the manufacturer's in- structions. Samples were loaded on 10\% SDS-polyacrylamide gels (Wako Pure Chemical Industries) and transferred to polyvinylidene fluoride (PVDF) membranes (Novex). Next, the membranes were washed with TBS-T (25 mM Tris- $\mathrm{HCl}, \mathrm{pH} 7.4,0.1 \mathrm{w} / \mathrm{v} \%$ Tween-20, $137 \mathrm{~mm} \mathrm{NaCl}, 2.68 \mathrm{~mm} \mathrm{KCl}$; Wako Pure Chemical Industries) and blocked with $4 \%$ skim milk powder (Wako Pure Chemical Industries) for $1-2 \mathrm{~h}$ at room temperature. Following blocking, the membranes were incubated with primary antibodies against NFATc1 (BioLegend), cathepsin K (Abcam plc, Cambridge, UK), and $\beta$-actin (Wako Pure Chemical Industries) overnight at $4^{\circ} \mathrm{C}$, and then incubated with horseradish peroxidase-conjugated secondary antibodies (Cell Signaling Technology, Tokyo, Japan) for $1 \mathrm{~h}$ at room temperature. The membranes were washed several times with TBS-T between the blocking and incubation steps at room temperature. The protein signals were developed with an enhanced chemiluminescence reagent (ImmunoStar ${ }^{\circledR}$ LD; Wako Pure Chemical Industries) using an Image Quant ${ }^{\mathrm{TM}}$ LAS 500 (GE Healthcare) according to the manufacturer's instructions.

\section{Statistical Analysis}

All statistical analyses were performed using Student's $t$ test. A value of $p<0.05$ was considered to be statistically significant. The 95\% confidence intervals were calculated using GraphPad Prism software.

\section{Results}

\section{Synergistic Increase in Activin A Expression in}

Fresh-RSC Stimulated with TGF- $\beta_{1}$ and other

Inflammatory Cytokines

Several studies have demonstrated that expression of activin $\mathrm{A}$ in human synovial fibroblasts is induced by inflammatory cytokines such as IL- $1 \beta, \mathrm{TNF} \alpha$, and TGF- $\beta_{1}$ 


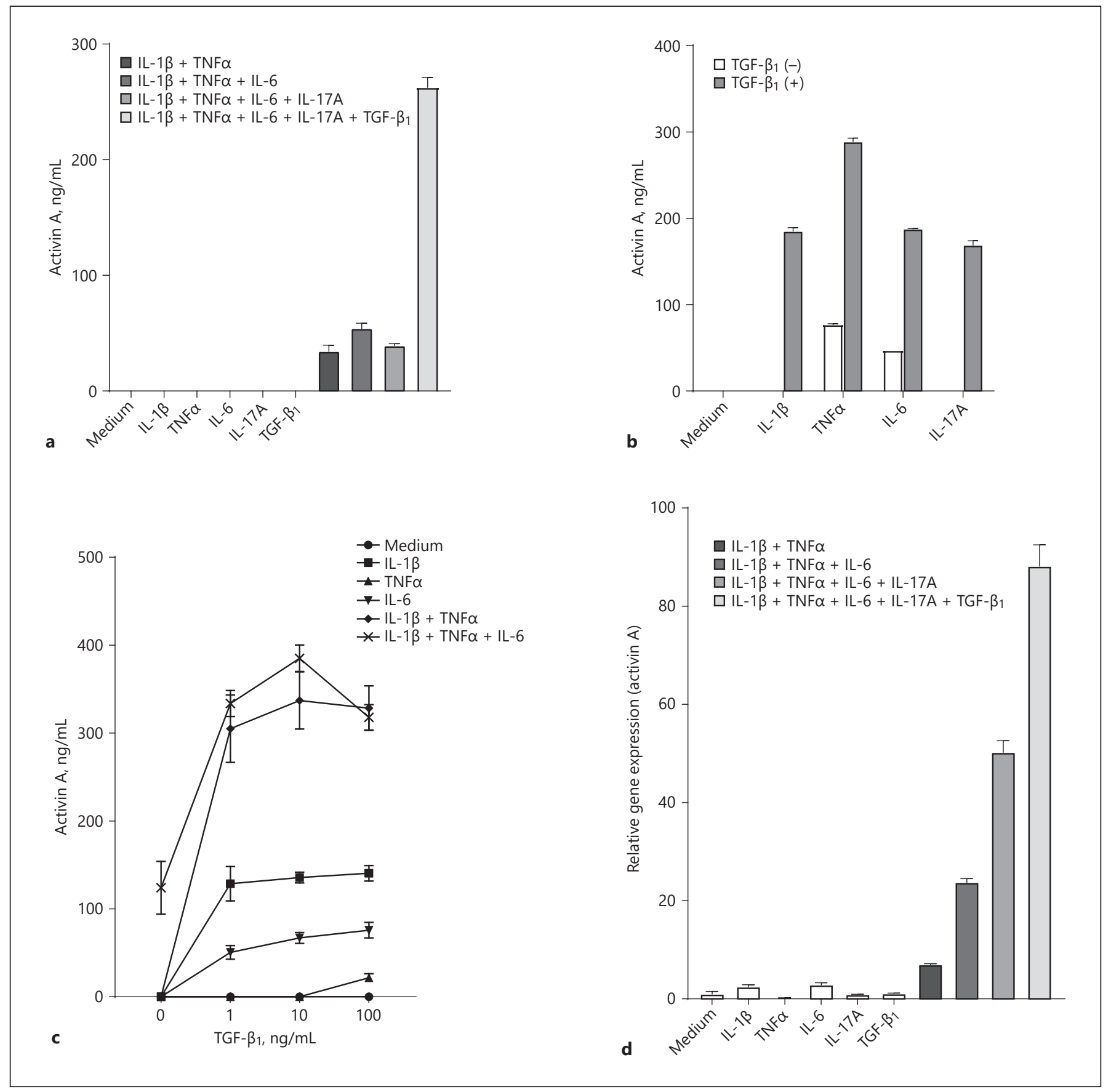

Fig. 1. Effect of proinflammatory cytokines (concentration: $50 \mathrm{ng} /$ $\mathrm{mL}$, respectively) on the production of activin A in freshly prepared rheumatoid synovial cells (fresh-RSC). a, b Fresh-RSC were cultured with IL- $1 \beta$, TNF $\alpha$, IL-6 with IL-6R $\alpha$, IL-17A, and TGF- $\beta_{1}$ either alone or in combination for $48 \mathrm{~h}$. $\mathrm{c}$ In the dose-dependent experiments, fresh-RSC were cultured with the indicated cytokines in the presence of increasing TGF- $\beta_{1}$ doses for $48 \mathrm{~h}$. Activin
A levels in the culture supernatant were determined by ELISA. d Fresh-RSC were cultured in the presence of the indicated cytokines either alone or in combination for $24 \mathrm{~h}$. Gene expression of activin $A$ was measured by quantitative PCR analysis. The data were normalized to those of $\beta$-actin. Results represent the mean \pm $\mathrm{SD}(n=3)$. 


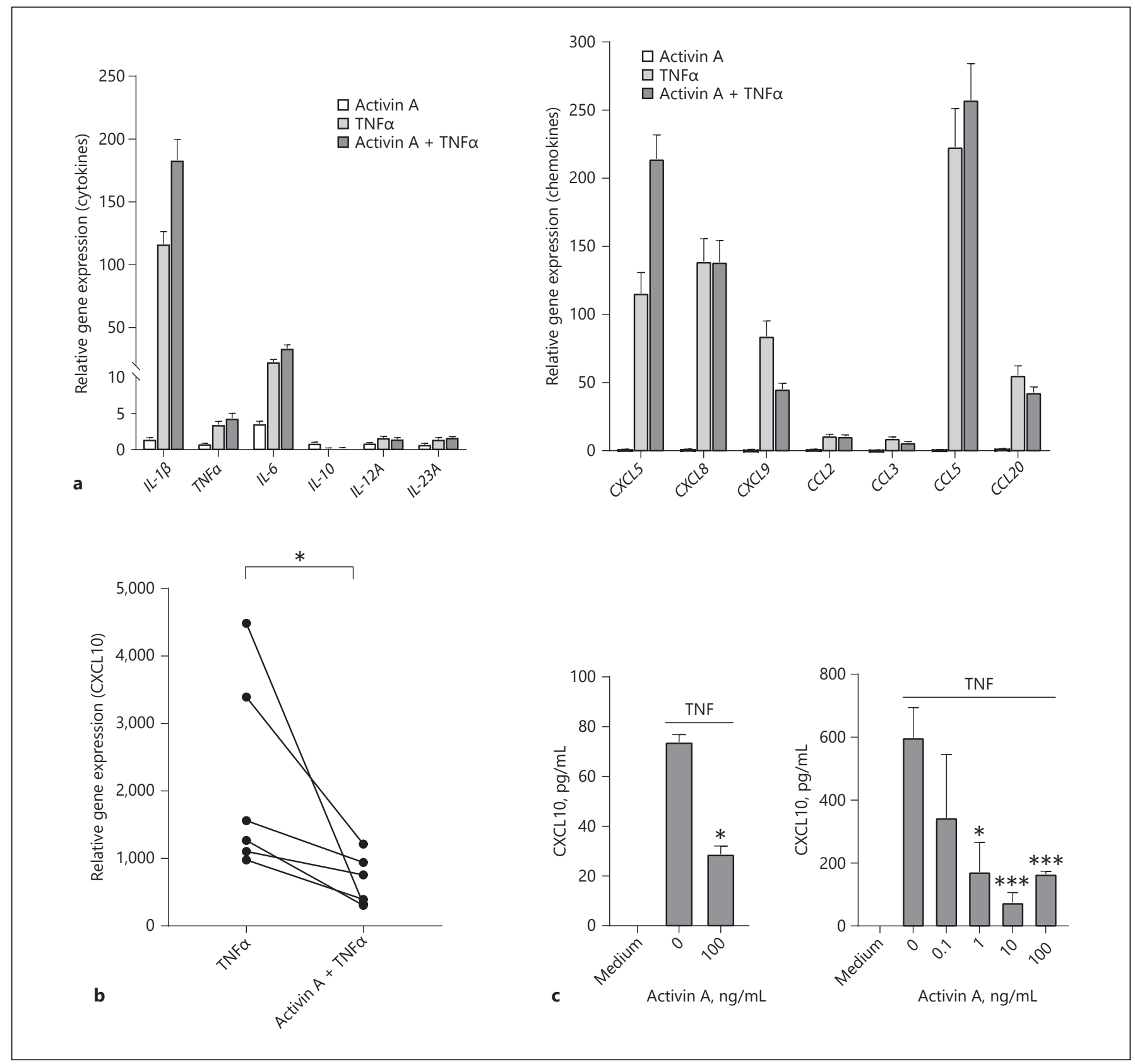

Fig. 2. Effect of activin A on the expression of inflammatory cytokines and chemokines with or without TNFa. a Freshly prepared rheumatoid synovial cells (fresh-RSC) were cultured with activin A (50 ng/mL) and/or TNFa (50 ng/mL) for $24 \mathrm{~h}$. Gene expression levels of inflammatory cytokines (IL-1 $\beta, T N F \alpha, I L-6, I L-10, I L-$ $12 A$, and $I L-23 A$ ) and chemokines (CXCL5, CXCL8, CXCL9, CCL2, CCL3, CCL5, and CCL20) were assessed and normalized to $\beta$-actin by quantitative PCR (qPCR) analysis. b Fresh-RSCs were cultured with TNFa and/or activin A for $24 \mathrm{~h}$. CXCL10 gene ex- pression was determined by qPCR analysis. Data represent 6 experiments conducted using synovial samples obtained from 4 patients with RA. ${ }^{*} p<0.05$. c Fresh-RSC were cultured with increasing doses of activin $A$ in the presence of TNFa $(50 \mathrm{ng} / \mathrm{mL})$ for $48 \mathrm{~h}$. After the culture, CXCL10 levels in cultured supernatants were determined using an ELISA kit $(\mathrm{R} \& \mathrm{D})$. Data represent the mean \pm SD from triplicate experiments. ${ }^{*} p<0.05,{ }^{* * *} p<0.001$, vs. TNFa. 


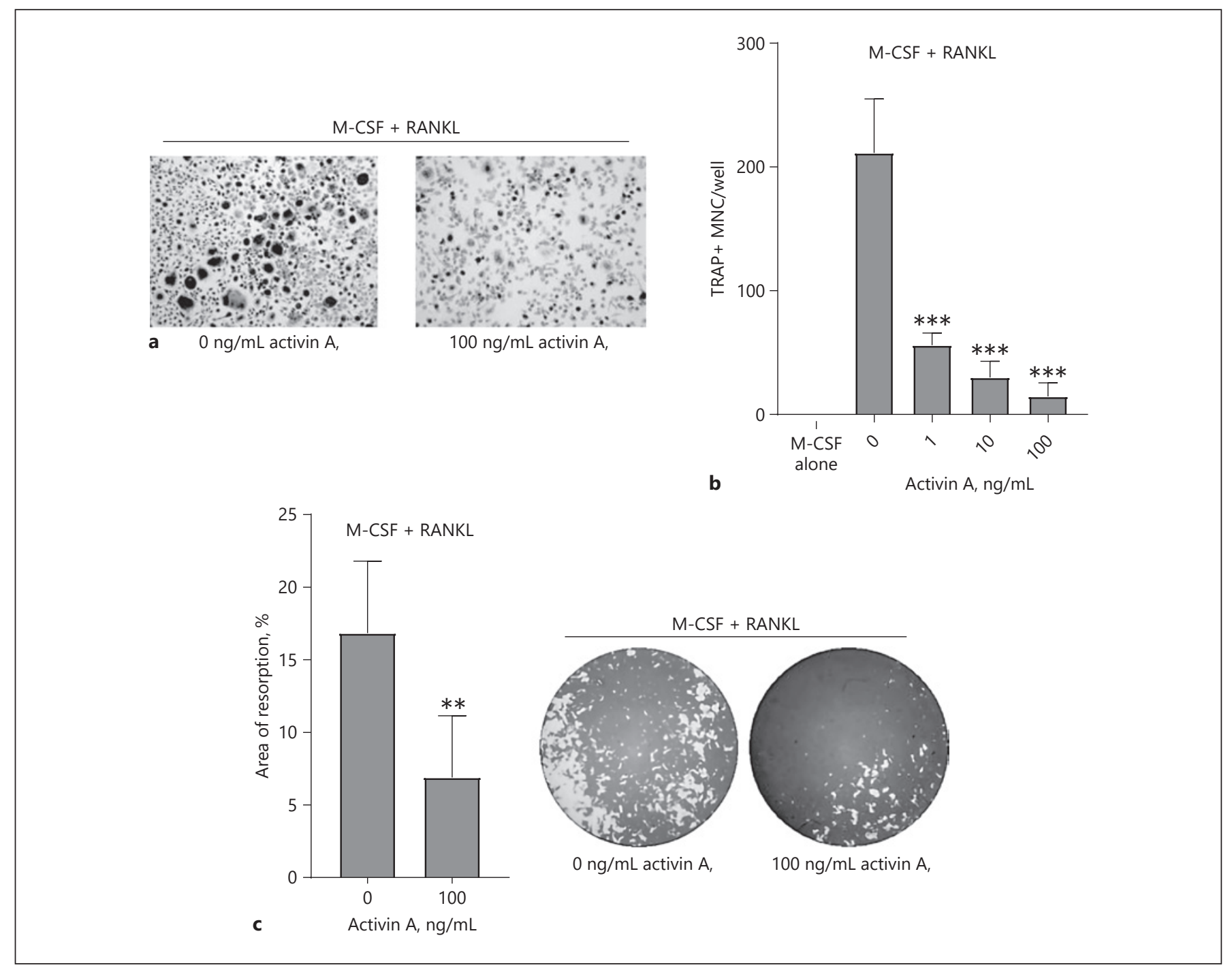

Fig. 3. Analysis of the effect of activin A on RANKL-induced osteoclast formation from human peripheral monocytes. a, b TRACP staining: human peripheral monocytes were cultured with M-CSF $(50 \mathrm{ng} / \mathrm{mL})$ in the presence of increasing doses of activin A for $24 \mathrm{~h}$, followed by the addition of RANKL (20 ng/mL). After 5-7 days, the cells were stained wit TRACP. c Bone resorption activity: human peripheral monocytes were cultured with M-CSF (50 ng/

[10]. However, the culture conditions and sources of fibroblasts and FLS varied among different studies, which might have complicated the interpretation of results. Therefore, we first examined the effect of proinflammatory cytokines on the expression of activin A in fresh-RSC from 3 patients with RA. The expression of an activin $A$ gene was quantified by qPCR, and protein concentrations in the cultured media were determined by ELISA. As shown in Figure $1 \mathrm{a}-\mathrm{d}$, the induction of activin A was
$\mathrm{mL})$ with or without activin $\mathrm{A}(20 \mathrm{ng} / \mathrm{mL})$ for $24 \mathrm{~h}$, followed by the addition of RANKL (20 ng/mL) on Osteo Assay Surface 96-well plates for 7-10 days. After culturing the cells, the adherent cells were lysed by osmotic shock and stained with $5 \%$ silver nitrate (Sigma-Aldrich) in PBS to quantify the resorption area using an EVOS microscope with ImageJ software. Data represent the mean \pm SD from triplicate experiments. ${ }^{* *} p<0.01,{ }^{* * *} p<0.001$. minimal in response to treatment with individual cytokines, including IL-1 $\beta$, TNFa, IL-6, IL-17A, and TGF- $\beta_{1}$, which were previously reported to induce activin A. In contrast, the combination of cytokines synergistically enhanced both the protein and mRNA expression levels of activin A (Fig. 1a, d). Notably, TGF- $\beta_{1}$ potently enhanced the production of activin A in combination with IL-1 $\beta$, TNFa, IL-6, and IL-17A (Fig. 1b). In our dose-response experiment, the effect of TGF- $\beta_{1}$ reached a plateau at a 


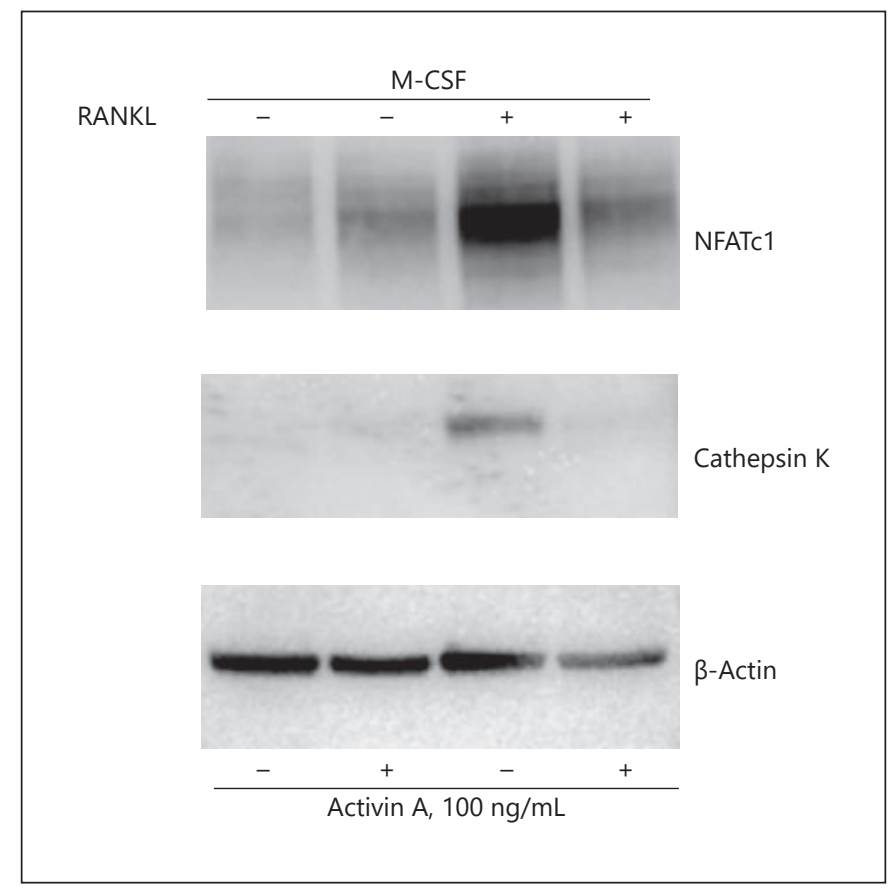

Fig. 4. Peripheral blood monocytes were cultured at a cell density of $2 \times 10^{5}$ cells/well in a 24 -well culture plate in the presence of MCSF $(50 \mathrm{ng} / \mathrm{mL})$ with or without activin A $(20 \mathrm{ng} / \mathrm{mL})$ for $24 \mathrm{~h}$, followed by the addition of RANKL $(50 \mathrm{ng} / \mathrm{mL})$. Following culture for $72 \mathrm{~h}$, protein expression of NFATcl and cathepsin K in the treated cells was determined by Western blotting. $\beta$-Actin protein levels were determined as a loading control.

concentration of $1 \mathrm{ng} / \mathrm{mL}$ (Fig. 1c). These data suggest that TGF- $\beta_{1}$ is the primary cytokine involved in activin A induction.

\section{Inhibition of TNF $\alpha$-Induced CXCL10 Expression by Activin A}

To clarify the role of activin A in RA inflammation, we next screened the expression of inflammatory cytokines (IL-1 $\beta$, TNFa, IL-6, IL-10, IL-12A, and IL-23A) and chemokines (CXCL5, CXCL8, CXCL9, CXCL10, CCL2, CCL3, CCL5, and CCL20) using qPCR analysis. As shown in Figure 2a, activin A did not stimulate RSC to induce these inflammatory cytokines. On the other hand, activin A significantly inhibited the TNFa-induced expression of CXCL10, a chemokine involved in chemoattraction of $\mathrm{T}$ cells and monocytes at both the protein and the mRNA level (Fig. 2b, c). This activin A-induced inhibition was consistently observed in fresh-RSC from 5 patients with RA (Fig. 2b). Dose-response analysis revealed that significant suppression was observed at the concentration of $1 \mathrm{ng} / \mathrm{mL}$ of activin A (Fig. 2c).

\section{Inhibitory Effect of Activin A on RANKL-Induced Osteoclast Generation in Human Peripheral Monocytes}

Although it has been shown that osteoclasts play an important role in joint destruction in RA, the role of activin A in osteoclastogenesis remains unclear. Therefore, we next evaluated the effect of activin A on RANKL-induced osteoclast formation from human monocytes. The resulting osteoclasts were identified with TRACP staining (Fig. 3a, b), and their bone resorption activity was assessed using Osteo plates (Fig. 3c). These data show that RANKL treatment induced osteoclast generation from PBMs, while activin A potently inhibited RANKL-mediated osteoclast generation. Notably, activin A-induced inhibition occurred in a dose-dependent manner (Fig. 3b). In addition, as shown in Figure 4, activin A potently inhibited the expression of RANKLinduced expression of NFATc1 (the critical signal molecule) and cathepsin K (a marker of osteoclastic differentiation).

\section{Discussion}

In the current study, we demonstrated that various inflammatory cytokines have either additive or synergistic effects on the expression of activin A in RSCs, and that TGF- $\beta_{1}$ is extremely important for the induction of activin A expression. Expression of activin A by FLS is stimulated by inflammatory cytokines such as TNFa, IL-1 $\beta$, and TGF- $\beta_{1}$, and IL- $1 \beta$ appears to be the strongest inducer of activin A secretion in FLS [10]. In contrast, our data showed that stimulation of these inflammatory cytokines alone resulted in minimal induction of activin A, which may be attributed to cell-type specific differences. Notably, fresh-RSC used in this study also contained CD14+ macrophages. To the best of our knowledge, this is the first study to report a strong synergistic relationship between TGF- $\beta_{1}$ and inflammatory cytokines such as IL$1 \beta$, TNF $\alpha$, and IL-6 in RSCs.

TGF- $\beta_{1}$, which is expressed in the rheumatoid synovium [24], is a major T-cell cytokine released from regulatory $\mathrm{T}$ cells $[25,26]$. A previous study reported potent induction of activin A secretion from human monocytes and bone marrow stromal fibroblasts by cognate interaction with activated T cells [27], suggesting the importance of T-cell cytokines for the induction of activin A. These data suggest that the induction of activin $\mathrm{A}$ in rheumatoid synovium may be related to the interaction of infiltrating T cells with synoviocytes via TGF- $\beta_{1}$. 
We reported for the first time that activin A suppressed TNFa-induced CXCL10 expression in rheumatoid synovium. In contrast, activin A did not affect the gene expression of inflammatory cytokines and chemokines. Consistent with the results of the current study, a number of previous studies have reported the anti-inflammatory actions of activin $\mathrm{A}$, such as activin $\mathrm{A}$-induced inhibition of IL- $1 \beta$ expression, IL- 6 activity [20], and matrix metalloproteinase-3 expression [17] in human tissues. In contrast, activin A has been reported to exert inflammatory actions by directing macrophage polarization toward the acquisition of an M1 proinflammatory phenotype [16]. In addition, CD14+ macrophages from RA synovial fluid exhibited an activin A-dependent proinflammatory profile [28]. These reports show the complexity of activin A actions in the inflammatory process. Further studies are needed to highlight the action of activin A in mediating inflammation in RA.

CXCL10 binds the receptor CXCR3 on $\mathrm{T}$ cells and Toll-like receptor 4 on monocytes and macrophages and upregulates immune responses through the activation and recruitment of $\mathrm{T}$ cells and monocytes [29-31]. CXCL10 has been detected in sera, synovial fluid, and tissues in RA patients [32,33]. Recently, CXCL10 has been reported to recruit inflammatory cells and be involved in bone erosion. For example, CXCL10 has been shown to recruit activated $\mathrm{T}$ cells which mediate osteoclastogenesis and recruit monocytes, which function as osteoclast precursors, to the inflamed joints [34]. Furthermore, in vivo treatment with antibodies against CXCL10 or its receptor attenuated bone destruction in mice with collagen-induced or adjuvant arthritis $[35,36]$. From these data, it is likely that activin A-induced inhibition of CXCL10 may lead to the prevention of bone destruction in RA.

Accumulation of activated osteoclasts at the inflamed joints plays a critical role in joint destruction in RA patients. Here, we demonstrated that activin A directly inhibited RANKL-induced osteoclast generation from human monocytes. Activin A also inhibited the protein expression of NFATc1 and cathepsin K. However, conflicting reports on the action of activin A on osteoclastogenesis have been published in the past. Fuller et al. [8] reported that activin A powerfully synergized with RANKL to induce osteoclast-like cells from murine bone marrow precursors, and osteoclast formation was prevented by exposure to soluble type IIA activin A receptor. Murase et al. [21] reported that the phosphorylation of p44/42 MAP kinase, p38 MAP kinase, and Smad2 is involved in activin A-enhanced osteoclast differentiation. In addition, activin $\mathrm{A}$ has been shown to enhance
RANK expression in osteoclast precursors [22]. In contrast, Fowler et al. [23] reported that activin A completely inhibited RANKL-stimulated osteoclast motility, differentiation, and bone resorption in stroma-free murine bone marrow macrophage cultures. Recently, Schoenmaker et al. [37] reported that activin A inhibits osteoclast formation stimulated with periodontal ligament fibroblasts from patients with fibrodysplasia ossificans progressiva, which is a genetic disease characterized by a mutation in the activin receptor type 1 ( $A C V R 1$ ) gene that results in enhanced responsiveness of the receptor to activin A. Moreover, consistent with our results, activin A inhibited RANKL-induced osteoclastogenesis in monocultures of CD14+ monocytes [37]. The discrepancy between the reported effects of activin A on osteoclastogenesis may be due to differences between species or culture systems.

For example, mouse bone marrow cells are used for osteoclastogenesis in murine experiments, but the possibility of contamination of adherent cells other than osteoclast precursor cells cannot be completely excluded. These cells may have modified the effects of activin A. On the other hand, activin A generally suppressed osteoclast differentiation in experiments using human monocytes, suggesting that the conflicting effects of activin A may be due to differences in species. Additional studies are needed to further clarify the mechanism of activin A action.

In summary, activin A production from fresh-RSC was markedly enhanced by a synergistic effect between TGF- $\beta_{1}$ and inflammatory cytokines. Activin A inhibited TNFa-induced CXCL10, an important chemoattractant for pathogenic-activated T cells and monocytes of osteoclast precursors, but it did not affect the expression of inflammatory cytokines and chemokines. In addition, activin A directly inhibited osteoclast formation in RA patients. These results suggest that activin A exerts an anti-inflammatory effect at inflamed joints to prevent joint destruction. Although additional studies are required to further elucidate this protective mechanism, our findings provide new insights into the role of activin A in protecting against joint destruction in RA patients. These findings may help to develop appropriate therapies for RA patients in the future.

\section{Acknowledgments}

The authors especially thank Dr. Yasunori Shimaoka (Hamawaki Orthopedic Hospital) for rheumatoid synovial specimens. 


\section{Statement of Ethics}

The study was approved by the Institutional Research and Ethics Committee of Hiroshima University (E-668-1), and written informed consent was obtained from all participants (healthy donors and patients with RA).

\section{Disclosure Statement}

The authors have no conflict of interest to declare.

\section{Funding Sources}

This work was supported by JSPS KAKENHI (grant No. 19K08908 to E.S., 19K18499 to S.M. and 19K07940 to S.H).

\section{Author Contributions}

E.S., S.H., S.M., and T.K.: conception and design of the study; E.S., T.K., K.O., T.T., K.Y., H.K., and Y.Y.: performing all experiments; E.S. and T.K.: drafting of the articles; E.S., S.H., S.M., and T.K.: critical revision of the articles for important intellectual content.

\section{References}

1 Huber LC, Distler O, Tarner I, Gay RE, Gay S, Pap T. Synovial fibroblasts: key players in rheumatoid arthritis. Rheumatology (Oxford). 2006 Jun;45(6):669-75.

2 Scott DL, Pugner K, Kaarela K, Doyle DV, Woolf A, Holmes J, et al. The links between joint damage and disability in rheumatoid arthritis. Rheumatology (Oxford). 2000 Feb; 39(2):122-32.

3 Bromley M, Woolley DE. Chondroclasts and osteoclasts at subchondral sites of erosion in the rheumatoid joint. Arthritis Rheum. 1984 Sep;27(9):968-75.

4 Goldring SR. Pathogenesis of bone erosions in rheumatoid arthritis. Curr Opin Rheumatol. 2002 Jul;14(4):406-10.

5 Goldring SR. Inflammatory mediators as essential elements in bone remodeling. Calcif Tissue Int. 2003 Aug;73(2):97-100.

6 Polzer K, Diarra D, Zwerina J, Schett G. Inflammation and destruction of the joints-the Wnt pathway. Joint Bone Spine. 2008 Mar; 75(2):105-7.

7 Bloise E, Ciarmela P, Dela Cruz C, Luisi S, Petraglia F, Reis FM. Activin A in mammalian physiology. Physiol Rev. 2019 Jan;99(1):739_ 80.

8 Fuller K, Bayley KE, Chambers TJ. Activin A is an essential cofactor for osteoclast induction. Biochem Biophys Res Commun. 2000 Feb;268(1):2-7.

9 Gribi R, Tanaka T, Harper-Summers R, Yu J. Expression of activin A in inflammatory arthropathies. Mol Cell Endocrinol. 2001 Jun; 180(1-2):163-7.

10 Ota F, Maeshima A, Yamashita S, Ikeuchi H, Kaneko Y, Kuroiwa T, et al. Activin A induces cell proliferation of fibroblast-like synoviocytes in rheumatoid arthritis. Arthritis Rheum. 2003 Sep;48(9):2442-9.

11 Shao L, Frigon NL Jr, Sehy DW, Yu AL, Lofgren J, Schwall R, et al. Regulation of production of activin $\mathrm{A}$ in human marrow stromal cells and monocytes. Exp Hematol. 1992 Nov; 20(10):1235-42.

12 El-Gendi SS, Moniem AE, Tawfik NM, Ashmawy MM, Mohammed OA, Mostafa AK, et al. Value of serum and synovial fluid activin $\mathrm{A}$ and inhibin $\mathrm{A}$ in some rheumatic diseases. Int J Rheum Dis. 2010 Aug;13(3):273-9.
13 Jones KL, Brauman JN, Groome NP, de Kretser DM, Phillips DJ. Activin A release into the circulation is an early event in systemic inflammation and precedes the release of follistatin. Endocrinology. 2000 May;141(5): 1905-8.

14 Jones KL, de Kretser DM, Clarke IJ, Scheerlinck JP, Phillips DJ. Characterisation of the rapid release of activin A following acute lipopolysaccharide challenge in the ewe. J Endocrinol. 2004 Jul;182(1):69-80.

15 Jones KL, Mansell A, Patella S, Scott BJ, Hedger MP, de Kretser DM, et al. Activin A is a critical component of the inflammatory response, and its binding protein, follistatin, reduces mortality in endotoxemia. Proc Nat Acad Sci USA. 2007 Oct;104(41):16239-44.

16 Sierra-Filardi E, Puig-Kröger A, Blanco FJ, Nieto C, Bragado R, Palomero MI, et al. Activin A skews macrophage polarization by promoting a proinflammatory phenotype and inhibiting the acquisition of anti-inflammatory macrophage markers. Blood. 2011 May;117(19):5092-101.

17 Chang DM, Liu SH, Lee HS, Lai JH, Chen CH. Activin A suppresses interleukin-1-induced matrix metalloproteinase 3 secretion in human chondrosarcoma cells. Rheumatol Int. 2007 Sep;27(11):1049-55.

18 Ohguchi M, Yamato K, Ishihara Y, Koide M, Ueda N, Okahashi N, et al. Activin A regulates the production of mature interleukin- $1 \beta$ and interleukin-1 receptor antagonist in human monocytic cells. J Interferon Cytokine Res. 1998 Jul;18(7):491-8.

19 Sugama S, Takenouchi T, Kitani H, Fujita M, Hashimoto M. Activin as an anti-inflammatory cytokine produced by microglia. J Neuroimmunol. 2007 Dec;192(1-2):31-9.

20 Yu EW, Dolter KE, Shao LE, Yu J. Suppression of IL- 6 biological activities by activin A and implications for inflammatory arthropathies. Clin Exp Immunol. 1998 Apr;112(1): 126-32.

21 Murase Y, Okahashi N, Koseki T, Itoh K, Udagawa N, Hashimoto O, et al. Possible involvement of protein kinases and Smad2 signaling pathways on osteoclast differentiation enhanced by activin A. J Cell Physiol. 2001 Aug;188(2):236-42.
22 Sugatani T, Alvarez UM, Hruska KA. Activin A stimulates I $\mathrm{KB}-\alpha / \mathrm{NF} \kappa \mathrm{B}$ and RANK expression for osteoclast differentiation, but not AKT survival pathway in osteoclast precursors. J Cell Biochem. 2003 Sep;90(1):59-67.

23 Fowler TW, Kamalakar A, Akel NS, Kurten RC, Suva LJ, Gaddy D. Activin A inhibits RANKL-mediated osteoclast formation, movement and function in murine bone marrow macrophage cultures. J Cell Sci. 2015 Feb; 128(4):683-94.

24 Szekanecz Z, Haines GK, Harlow LA, Shah MR, Fong TW, Fu R, et al. Increased synovial expression of transforming growth factor (TGF) $-\beta$ receptor endoglin and TGF- $\beta 1$ in rheumatoid arthritis: possible interactions in the pathogenesis of the disease. Clin Immunol Immunopathol. 1995 Aug;76(2):187-94.

25 Kehrl JH, Wakefield LM, Roberts AB, Jakowlew S, Alvarez-Mon M, Derynck R, et al. Production of transforming growth factor beta by human $\mathrm{T}$ lymphocytes and its potential role in the regulation of $\mathrm{T}$ cell growth. J Exp Med. 1986 May;163(5):1037-50.

26 Seeger P, Bosisio D, Parolini S, Badolato R, Gismondi A, Santoni A, et al. Activin A as a mediator of NK-dendritic cell functional interactions. J Immunol. 2014 Feb;192(3): 1241-8.

27 Abe M, Shintani Y, Eto Y, Harada K, Kosaka $\mathrm{M}$, Matsumoto T. Potent induction of activin A secretion from monocytes and bone marrow stromal fibroblasts by cognate interaction with activated T cells. J Leukoc Biol. 2002 Aug;72(2):347-52.

28 Soler Palacios B, Estrada-Capetillo L, Izquierdo E, Criado G, Nieto C, Municio C, et al. Macrophages from the synovium of active rheumatoid arthritis exhibit an activin A-dependent pro-inflammatory profile. J Pathol. 2015 Feb;235(3):515-26.

29 Lee EY, Lee ZH, Song YW. CXCL10 and autoimmune diseases. Autoimmun Rev. 2009 Mar;8(5):379-83.

30 Rotondi M, Chiovato L, Romagnani S, Serio $\mathrm{M}$, Romagnani P. Role of chemokines in endocrine autoimmune diseases. Endocr Rev. 2007 Aug;28(5):492-520. 
31 Schulthess FT, Paroni F, Sauter NS, Shu L, Ribaux P, Haataja L, et al. CXCL10 impairs $\beta$ cell function and viability in diabetes through TLR4 signaling. Cell Metab. 2009 Feb;9(2): 125-39.

32 Hanaoka R, Kasama T, Muramatsu M, Yajima N, Shiozawa F, Miwa Y, et al. A novel mechanism for the regulation of IFN-gamma inducible protein-10 expression in rheumatoid arthritis. Arthritis Res Ther. 2003;5(2): R74-81.
33 Patel DD, Zachariah JP, Whichard LP. CXCR3 and CCR5 ligands in rheumatoid arthritis synovium. Clin Immunol. 2001 Jan; 98(1):39-45.

34 Lee JH, Kim B, Jin WJ, Kim HH, Ha H, Lee $\mathrm{ZH}$. Pathogenic roles of CXCL10 signaling through CXCR3 and TLR4 in macrophages and T cells: relevance for arthritis. Arthritis Res Ther. 2017 Jul;19(1):163.

35 Kwak HB, Ha H, Kim HN, Lee JH, Kim HS, Lee $S$, et al. Reciprocal cross-talk between RANKL and interferon-gamma-inducible protein 10 is responsible for bone-erosive experimental arthritis. Arthritis Rheum. 2008 May;58(5):1332-42.
36 Mohan K, Issekutz TB. Blockade of chemokine receptor CXCR3 inhibits $\mathrm{T}$ cell recruitment to inflamed joints and decreases the severity of adjuvant arthritis. J Immunol. 2007 Dec;179(12):8463-9.

37 Schoenmaker T, Wouters F, Micha D, Forouzanfar T, Netelenbos C, EekhoffEM, et al. The effect of Activin-A on periodontal ligament fibroblasts-mediated osteoclast formation in healthy donors and in patients with fibrodysplasia ossificans progressiva. J Cell Physiol. 2019 Jul;234(7):10238-47. 